\title{
Seeking the balance: patching double and exceptional field theories
}

\section{G. Papadopoulos}

Department of Mathematics, King's College London, Stand, London WC2R 2LS, U.K.

E-mail: george.papadopoulos@kcl.ac.uk

ABstract: We investigate the patching of double and exceptional field theories. In double field theory the patching conditions imposed on the spacetime after solving the strong section condition imply that the 3 -form field strength $H$ is exact. A similar conclusion can be reached for the form field strengths of exceptional field theories after some plausive assumptions are made on the relation between the transition functions of the additional coordinates and the patching data of the form field strengths. We illustrate the issues that arise, and explore several alternative options which include the introduction of C-folds and of the topological geometrisation condition.

KeYwords: String Duality, Differential and Algebraic Geometry, M-Theory

ARXiv EPrint: 1402.2586 


\section{Contents}

1 Introduction 1

2 A review of $\mathrm{U}(1)$ field paradigm 3

3 Patching 3-forms and double coordinates 5

3.1 Transition functions of closed 3-forms 5

$\begin{array}{ll}3.2 & \text { Transition functions and exact 3-forms }\end{array}$

3.3 Patching DFT 6

3.4 Seeking a consistent patching 8

4 Revisiting examples $\quad 9$

4.1 Reviewing the double space of 3-torus with constant $H$ flux $\quad 9$

4.2 Patching the double space of 3-torus with constant $H \quad 10$

$\begin{array}{ll}\text { 4.2.1 Patching conditions } & 10\end{array}$

$\begin{array}{lll}\text { 4.2.2 Consistency of the patching } & 12\end{array}$

5 Patching k-forms and exceptional coordinates 13

$\begin{array}{ll}5.1 \text { Transition functions of closed k-forms } & 13\end{array}$

$\begin{array}{ll}5.2 \text { Exact k-forms and patching conditions } & 14\end{array}$

$\begin{array}{ll}\text { 5.3 Seeking a consistent patching for EFT } & 14\end{array}$

$\begin{array}{lll}6 & \text { Summary and outlook } & 15\end{array}$

A Modifying transition functions $\quad 18$

$\begin{array}{ll}\text { A.1 New transition functions and k-forms } & 18\end{array}$

$\begin{array}{ll}\text { A.2 Testing for other properties } & 19\end{array}$

$\begin{array}{lll}\text { A.3 } \tilde{M} \text { for M-theory } & 20\end{array}$

\section{Introduction}

It has been known for sometime that given a spacetime $M$ and a 2 -form gauge field, $F$, $d F=0$, one can introduce an additional coordinate, a charge coordinate, and together with the Dirac quantisation condition, one can construct a U(1) bundle $M_{F}$ over the spacetime $M$. This bundle is a new manifold associated to the Maxwell field and the transition functions of the fibre are related to the patching conditions of the 1-form gauge potential of $F$. This construction is a manifestation of the isomorphism of $\mathrm{U}(1)$ bundles over $M$ with the cohomology classes in $H^{2}(M, \mathbb{Z})$ and underpins Kaluza-Klein theory. In the latter, the $\mathrm{U}(1)$ gauge potential is a component of the metric in higher dimensions. This is sometimes referred as the geometrisation ${ }^{1}$ of the $\mathrm{U}(1)$ field. A feature of the construction is that the charge of the $\mathrm{U}(1)$ field is replaced by information stored in the patching conditions of $M_{F}$.

\footnotetext{
${ }^{1}$ Later, we shall introduce a related notion referred to as "topological geometrisation".
} 
Similar suggestions have emerged in the context of string theory and M-theory following the early works of [1-4]. These include double field theory (DFT) of [5-10] applied to string theory, the $E_{11}[11,12]$, and the exceptional field theory (EFT) [13-19] proposals, see also reviews [20-22] and references within. There are several reasons for this. One is to find a geometric realization of string and M-theory dualities and to describe duality covariant theories. Another is to explore the idea that string and M-theory dualities emerge as symmetries of the 10- and 11-dimensional theories rather than just their toroidal compactifications following the early work of [23]. The constructions are broadly based on a similar technology to the 2-form U(1) gauge field described above but now the metric in higher dimensions is replaced by a generalized metric which includes the form gauge potentials of string theory and M-theory, and the introduction of suitable new coordinates.

A related construction is that of generalized geometry [24-26]. In generalized geometry no new coordinates are introduced in addition to those of spacetime. Instead the tangent space of the spacetime is replaced by a vector bundle $E$ which is an extension of $T M$ equipped with an appropriate bracket. Such an approach has been used to explore some geometric properties of supergravities associated with strings and M-theory [27-30].

Although much work has been done to understand the geometry that underpins DFT and EFT several questions remain. One question is to unravel topological and differential structures of double and exceptional spaces, and another related question is to understand how information about string and brane charges is stored in their topology. In the context of the formalism developed so far, it is not possible to answer these questions because most of the computations have been made using infinitesimal transformations generated by generalized Lie derivatives. However for DFT a set of finite transformations have been proposed $[22,31]$ by integrating the infinitesimal transformations that have been known before, and have been explored as transition functions for double spaces in [32]. Infinitesimal transformations for the coordinates of exceptional spaces underlying EFT have been proposed in e.g. [13-15, 17, 18, 33, 34].

In this paper, we shall review some of the properties of patching closed form field strengths on a spacetime and illustrate the issues involved. Then we shall demonstrate that the transformations of $[22,31]$ that solve the strong section condition after interpreting them as patching conditions following [32] imply that the NS-NS 3-form field strength $H$ which arises in string theory is an exact 3 -form.

In addition, we explore the double space of $T^{3}$ with H-flux background of [22] from the patching conditions point of view. We find that without additional assumptions on the patching conditions, the construction leads to an inconsistency as it requires that $H$ is exact. However, we also show that it is possible to modify the patching conditions at triple overlaps in such a way that consistency is restored. But such a modification is dependent on the existence of a particular atlas on $T^{3}$, i.e. it is not general covariant, and so it cannot be adapted to other backgrounds.

We shall further argue that a similar conclusion can be reached in the context of EFT provided that the patching conditions of closed form field strengths are related in a linear way to the transition functions of the additional coordinates of the exceptional spaces and the combinatorial law follows the usual rules of tensor calculus. The latter point will 
be illustrated in the $\mathrm{U}(1)$ paradigm reviewed in the next section. Concerns about the consistency of patching EFT have also been raised in [37] using the Cartan theorem [38].

We shall explore several alternative ways to reconcile the transition functions of the double and exceptional spaces with the patching conditions of the closed form field strengths on the spacetime. We shall see that there are examples described in appendix A, where this can be done at a cost. In particular, these constructions do not exhibit some key properties of the $\mathrm{U}(1)$ paradigm. Nevertheless, they illustrate some of the issues involved and provide a local model of a consistent construction.

In the conclusions, we propose a general scheme with a minimum number of requirements that should be followed in order to construct double and exceptional spaces which exhibit the key properties of the U(1) paradigm and allow for a realization of duality groups. To distinguish them from previous constructions, we shall call these new spaces "C-folds", i.e. manifolds with charge coordinates. One of the key requirements that we propose is the "topological geometrisation condition". This states that the pull back on the C-fold of the closed form field strengths on a spacetime must be exact. One of the consequences of this condition is that the fibres of the C-fold must have a non-trivial topology. We propose several constructions for C-folds based on K-theory and homotopy theory.

This paper has been organized as follows. In section 2, we describe the U(1) field paradigm. In section 3, we show that for DFT the patching conditions allow for only exact form field strengths. I section 4, we revisit the construction of the double space of $T^{3}$ with $H$-flux background. In section 5 , we show under certain assumptions a similar statement for the EFT. In section 6, we give our conclusions and explore some future directions. In appendix A, we give an example of an alternative construction for double and exceptional geometries consistent with closed form field strengths.

\section{A review of $\mathrm{U}(1)$ field paradigm}

Before we proceed to investigate how to extend a spacetime with additional coordinates associated to a general closed $\mathrm{k}$-form, it is instructive to revisit the construction for $\mathrm{U}(1)$ fields which is well known.

To begin, suppose $M$ is a n-dimensional manifold equipped with a good cover $\left\{U_{\alpha}\right\}_{\alpha \in I}$, see e.g. [41], and a closed 2-form $\omega^{2}$. On each open set $U_{\alpha}$, the Poincaré theorem implies that there are 1-form potentials $C_{\alpha}^{1}$ such that

$$
\omega_{\alpha}^{2}=d C_{\alpha}^{1} .
$$

Using repeatedly the Poincaré lemma at double and triple overlaps $U_{\alpha} \cap U_{\beta}$ and $U_{\alpha} \cap U_{\beta} \cap U_{\gamma}$, one has that

$$
C_{\alpha}^{1}-C_{\beta}^{1}=d a_{\alpha \beta}^{0}, \quad a_{\alpha \beta}^{0}+a_{\gamma \alpha}^{0}+a_{\beta \gamma}^{0}=n_{\alpha \beta \gamma},
$$

where $n_{\alpha \beta \gamma}$ are constants. Now if $\omega^{2}$ represents a class in $H^{2}(M, \mathbb{Z})$, then $n_{\alpha \beta \gamma} \in 2 \pi \mathbb{Z}$ are integers.

The geometrisation of the U(1) field proceeds as follows. Starting from the good cover $\left\{U_{\alpha}\right\}_{\alpha \in I}$ of $M$, one introduces a new space with charts $U_{\alpha} \times \mathbb{R}$, and transition functions

$$
x_{\alpha}=f_{\alpha \beta}\left(x_{\beta}\right), \quad \theta_{\alpha}=\theta_{\beta}+a_{\alpha \beta}^{0},
$$


where $x_{\alpha}$ and $f_{\alpha \beta}$ are the coordinates and transition functions of $M$ and $\theta_{\alpha}$ is an additional coordinate. At first sight it appears that the second transition function is consistent with (2.2) at triple overlaps iff $n_{\alpha \beta \gamma}=0$. As we shall prove later, this implies that $\omega^{2}$ is exact. However, for any closed $\omega^{2}$ which represents a class in $H^{2}(M, \mathbb{Z})$, the transition functions are consistent at triple overlaps by taking the addition in the second transition function in $(2.3)$ to be over $\bmod 2 \pi \mathbb{Z}$, i.e. the new transition functions are

$$
x_{\alpha}=f_{\alpha \beta}\left(x_{\beta}\right), \quad\left(\theta_{\alpha}-\theta_{\beta}-a_{\alpha \beta}^{0}\right)=0 \bmod 2 \pi \mathbb{Z} .
$$

In such a case no further condition arises as the sum over $a^{0}$ 's in (2.2) at triple overlaps vanishes $\bmod 2 \pi \mathbb{Z}$.

The effect of this construction is to define a circle bundle $M_{\omega^{2}}$ over $M$ with transition functions $\phi_{\alpha \beta}=\exp \left(i a_{\alpha \beta}^{0}\right)$. Some of the features and consequences of this construction are as follows.

(i) An integral part of the construction is the modification of the combinatorial law that it is used to describe the transition functions of the additional coordinate. This is related to the requirement that $\omega^{2}$ represents a class in $H^{2}(M, \mathbb{Z})$.

(ii) The topological structure of $M_{\omega^{2}}$ is completely determined by the class $\left[\omega^{2}\right]$ of $\omega^{2}$ in $H^{2}(M, \mathbb{Z})$, i.e. $M_{\omega^{2}}=M_{\left[\omega^{2}\right]}$, and vice versa, as there is a 1-1 correspondence between circle bundles and elements of $H^{2}(M, \mathbb{Z})$.

(iii) The tangent bundle $T M_{\omega^{2}}$ of $M_{\omega^{2}}$ is an extension of $T M$ with respect to a trivial real line bundle $L$, i.e.

$$
0 \rightarrow L \rightarrow T M_{\omega^{2}} \rightarrow \pi^{*} T M \rightarrow 0
$$

where $\pi: M_{\omega^{2}} \rightarrow M$ is a projection.

(iv) Another feature of the construction is that $\pi^{*} \omega^{2}$ is an exact form on $M_{\omega^{2}}$ as

$$
\pi^{*} \omega^{2}=-d\left(d \theta-\pi^{*} C^{1}\right)
$$

and $\left(d \theta-\pi^{*} C^{1}\right)$ is a globally defined 1-form on $M_{\omega^{2}}$.

The last property can be seen as the "topological geometrisation" of $\omega^{2}$. On $M, \omega^{2}$ has charges which are given by the periods

$$
n_{i}=\int_{B_{i}} \omega^{2}
$$

where $\left(B_{i}\right)$ is a basis in $H_{2}(M, \mathbb{Z})$. Since $\pi^{*} \omega^{2}$ is exact on $M_{\omega^{2}}$, all the periods of $\pi^{*} \omega^{2}$ on $M_{\omega^{2}}$ vanish. Instead all the information carried by the periods $n_{i}$ has been replaced by the topology of $M_{\omega^{2}}$.

The above construction can be generalized to include more than one U(1) field strengths leading to toric fibrations over $M$. In addition this can be generalized to non-abelian gauge fields which in turn leads to principal bundles with fibre the gauge group. In both cases all the properties mentioned above, with some minor modifications, apply to the more general set up. 


\section{Patching 3-forms and double coordinates}

\subsection{Transition functions of closed 3 -forms}

Before, we proceed to the patching of $\mathrm{k}$-forms and the introduction of new coordinates, it is instructive to explain the patching of closed 3-forms $\omega^{3}$. For this let $M$ be a n-dimensional manifold $^{2}$ with a good cover $\left\{U_{\alpha}\right\}_{\alpha \in I}$ and a partition of unity $\left\{\rho_{\alpha}\right\}_{\alpha \in I}$ subordinate to $\left\{U_{\alpha}\right\}_{\alpha \in I}$. This means that $M$ admits functions $\rho_{\alpha} \geq 0$ with support in $U_{\alpha}$ such that at every point $x \in M$

$$
\sum_{\alpha} \rho_{\alpha}=1
$$

where the sum is taken over a finite collection. For a discussion on partitions of unity and the different kinds that exist see [41]. Here we shall use those partitions of unity that have the same index set $I$ as that of the good cover and so they do not have necessarily compact support.

To continue, take $\omega^{3}$ to be a closed 3 -form on $M$. Using the Poincaré lemma at each open set $U_{\alpha}$, we can write $\omega^{3}$ in terms of a gauge potential $C$ as

$$
\omega_{\alpha}^{3}=d C_{\alpha}^{2} .
$$

Using repeatedly the Poincaré lemma at the double, $U_{\alpha} \cap U_{\beta}$, triple, $U_{\alpha} \cap U_{\beta} \cap U_{\gamma}$, and quadruple, $U_{\alpha} \cap U_{\beta} \cap U_{\gamma} \cap U_{\delta}$, overlaps, we have

$$
C_{\alpha}^{2}=C_{\beta}^{2}+d a_{\alpha \beta}^{1}, \quad a_{\alpha \beta}^{1}+a_{\beta \gamma}^{1}+a_{\gamma \alpha}^{1}=d a_{\alpha \beta \gamma}^{0}, \quad a_{\alpha \beta \gamma}^{0}-a_{\delta \alpha \beta}^{0}+a_{\gamma \delta \alpha}^{0}-a_{\beta \gamma \delta}^{0}=n_{\alpha \beta \gamma \delta},
$$

where $n_{\alpha \beta \gamma \delta}$ are constants. Note that the patching data $a_{\alpha \beta}^{1}, a_{\alpha \beta \gamma}^{0}, n_{\alpha \beta \gamma \delta}$ are skewsymmetric in the interchange of any two of the open set labels, i.e. $a_{\beta \gamma}^{1}=-a_{\gamma \beta}^{1}$ and similarly for the rest. Moreover $n_{\alpha \beta \gamma \delta}$ are restricted to be multiples of integers if $\omega^{k}$ represents a class in $H^{3}(M, \mathbb{Z})$. In particular, the patching condition of 2-form gauge potential in local coordinates reads

$$
\begin{aligned}
\left(C_{\alpha}^{2}\right)_{i j} & =\frac{\partial x_{\beta}^{k}}{\partial x_{\alpha}^{i}} \frac{\partial x_{\beta}^{l}}{\partial x_{\alpha}^{j}}\left(C_{\beta}^{2}\right)_{k l}+\frac{\partial}{\partial x_{\alpha}^{i}} a_{\alpha \beta, j}^{1}-\frac{\partial}{\partial x_{\alpha}^{j}} a_{\alpha \beta, i}^{1} \\
& =\frac{\partial x_{\beta}^{k}}{\partial x_{\alpha}^{i}} \frac{\partial x_{\beta}^{l}}{\partial x_{\alpha}^{j}}\left(\left(C_{\beta}^{2}\right)_{k l}+\frac{\partial}{\partial x_{\beta}^{k}} a_{\alpha \beta, l}^{1}-\frac{\partial}{\partial x_{\beta}^{l}} a_{\alpha \beta, k}^{1}\right) .
\end{aligned}
$$

Observe that the choice of gauge potentials is not unique. In particular, they are defined up to the gauge transformation

$$
C_{\alpha}^{2} \rightarrow C_{\alpha}^{2}+d \chi_{\alpha}^{1}
$$

Similarly, the patching data $a_{\beta \gamma}^{1}$ of $\omega^{3}$ at double overlaps are not uniquely defined either. In particular, they are defined up to a gauge transformation

$$
a_{\alpha \beta}^{1} \rightarrow a_{\alpha \beta}^{1}-\chi_{\alpha}^{1}+\chi_{\beta}^{2}+d \psi_{\alpha \beta}^{0},
$$

\footnotetext{
${ }^{2}$ In what follows, the signature of $M$ is not essential and the results apply to both Riemannian and Lorentzian manifolds.
} 
for a 0 -form $\psi_{\alpha \beta}^{0}$ defined on double overlaps. This is the only ambiguity that one has in determining the patching conditions on double overlaps of $\omega^{3}$. Anything else is inconsistent with the identification of $\omega^{3}$ as a closed 3-form on $M$.

\subsection{Transition functions and exact 3 -forms}

Before we proceed to compare the above patching conditions of closed 3-forms with those that arise in DFT, we shall prove a technical lemma which arises in the context of Čechde Rham theory. In particular, if there is a choice of patching data, up to (3.6) gauge transformations, such that $a_{\alpha \beta}^{1}$ on triple overlaps $U_{\alpha} \cap U_{\beta} \cap U_{\gamma}$ satisfies the cocycle ${ }^{3}$ condition

$$
a_{\alpha \beta}^{1}+a_{\beta \gamma}^{1}+a_{\gamma \alpha}^{1}=0
$$

then $\omega^{3}$ is exact.

For this suffices to show that $\omega^{3}=d \tilde{C}^{2}$, where $\tilde{C}^{2}$ is a 2 -form on $M$, i.e. $\tilde{C}_{\alpha}=\tilde{C}_{\beta}$. Indeed define

$$
\tilde{C}_{\alpha}^{2}=C_{\alpha}^{2}+\Sigma_{\gamma} d\left(\rho_{\gamma} a_{\gamma \alpha}^{1}\right) .
$$

Observe that $d \tilde{C}_{\alpha}=d C_{\alpha}=\omega^{3}$ and

$$
\tilde{C}_{\alpha}^{2}-\tilde{C}_{\beta}^{2}=C_{\alpha}^{2}-C_{\beta}^{2}+\Sigma_{\gamma} d\left(\rho_{\gamma} a_{\gamma \alpha}^{1}\right)-\Sigma_{\gamma} d\left(\rho_{\gamma} a_{\gamma \beta}^{1}\right)=d a_{\alpha \beta}^{1}-\Sigma_{\gamma} d\left(\rho_{\gamma} a_{\alpha \beta}^{1}\right)=d a_{\alpha \beta}^{1}-d a_{\alpha \beta}^{1}=0
$$

where to establish the last two equalities we have used (3.7) and (3.1), respectively. Thus if (3.7) holds, $\omega^{3}$ is exact and represents the trivial class in $H^{3}(M, \mathbb{R})$.

\subsection{Patching DFT}

After integrating the infinitesimal transformations generated by generalized Lie derivatives, generalized finite tensor transformations have been proposed for $\mathrm{DFT}^{4}$ in $[22,31]$ and further explored as transition functions in [32]. According to these generalized tensor transformations, 1-forms are transformed as

$$
\omega_{N}^{\prime}=F_{N}^{M} \omega_{M}
$$

where

$$
F_{N}^{M}=\frac{1}{2}\left(\frac{\partial X^{P}}{\partial X^{\prime N}} \frac{\partial X_{P}^{\prime}}{\partial X_{M}}+\frac{\partial X_{N}^{\prime}}{\partial X_{P}} \frac{\partial X^{M}}{\partial X^{\prime P}}\right)
$$

and where $X^{M}$ are the coordinates of the double space and indices are raised and lowered with the split metric $\eta_{M N}$ and $X^{\prime M}=X^{\prime M}\left(X^{N}\right)$. All the fields and coordinate transformations satisfy the strong section condition.

Viewing the above transformations as patching conditions and putting them into the language of the previous section, we have

$$
X_{\alpha}^{M}=X_{\alpha \beta}^{M}\left(X_{\beta}^{N}\right),\left(\omega_{\alpha}\right)_{N}=\left(F_{\alpha \beta}\right)_{N}^{M}\left(\omega_{\beta}\right)_{M},
$$

\footnotetext{
${ }^{3}$ This is in the sense of Čech cohomology, see [41].

${ }^{4}$ For some mathematical aspects of DFT see e.g. [35] and also [36] for explicit backgrounds.
} 
where

$$
\left(F_{\alpha \beta}\right)_{N}{ }^{M}=\frac{1}{2}\left(\frac{\partial X_{\beta}^{P}}{\partial X_{\alpha}^{N}} \frac{\partial X_{\alpha P}}{\partial X_{\beta M}}+\frac{\partial X_{\alpha N}}{\partial X_{\beta P}} \frac{\partial X_{\beta}^{M}}{\partial X_{\alpha}^{P}}\right) .
$$

An extensive investigation of the transformations induced on the fields after solving the strong section condition ${ }^{5}$ has been made in $[22,31]$. Interpreting these transformations as patching conditions, one can show that if one considers either

$$
x_{\alpha}^{i}=x_{\alpha \beta}^{i}\left(x_{\beta}^{j}\right), \quad y_{\alpha i}=y_{\beta i}
$$

or shift transformations

$$
x_{\alpha}^{i}=x_{\beta}^{i}, \quad y_{\alpha i}=y_{\beta i}-\zeta_{\alpha \beta i}
$$

of the double coordinates, then the 2-form gauge potential $b$ of the NS-NS 3-form field strength transforms either as a 2-form, $b_{\alpha}=b_{\beta}$ or as $b_{\alpha}=b_{\beta}+d \zeta_{\alpha \beta}$, where in the latter case the $x$ transformation is the identity

Clearly, none of these two patching conditions are satisfactory. If $b$ transforms as a 2-form, then one has to restrict $H$ to be exact. The shift transformation is also not sufficient as we know that string theory admits solutions which require that $M$ patches with non-trivial transition functions.

The authors of [22] have also investigated the combined transformation which includes diffeomorphisms of the spacetime $M$ combined with shift transformations of the $y$ coordinates which as a patching condition reads

$$
x_{\alpha}^{i}=x_{\alpha \beta}^{i}\left(x_{\beta}^{j}\right), \quad y_{\alpha i}=y_{\beta i}-\zeta_{\alpha \beta i} .
$$

In turn these give rise to the patching condition

$$
\begin{aligned}
\left(b_{\alpha}\right)_{i j}= & \frac{\partial x_{\beta}^{k}}{\partial x_{\alpha}^{i}} \frac{\partial x_{\beta}^{l}}{\partial x_{\alpha}^{j}}\left(\left(b_{\beta}\right)_{k l}+\frac{1}{2}\left(\frac{\partial}{\partial x_{\beta}^{k}} \zeta_{\alpha \beta, l}-\frac{\partial}{\partial x_{\beta}^{l}} \zeta_{\alpha \beta, k}\right)\right) \\
& +\frac{1}{2}\left(\frac{\partial x_{\beta}^{k}}{\partial x_{\alpha}^{i}} \frac{\partial}{\partial x_{\beta}^{k}} \zeta_{\alpha \beta, j}-\frac{\partial x_{\beta}^{k}}{\partial x_{\alpha}^{j}} \frac{\partial}{\partial x_{\beta}^{k}} \zeta_{\alpha \beta, i}\right)
\end{aligned}
$$

for the 2-form gauge potential $b$.

It is clear from (3.16) that consistency on triple overlaps on the spacetime requires that

$$
\zeta_{\alpha \beta}+\zeta_{\beta \gamma}+\zeta_{\gamma \alpha}=0
$$

If this is the case, a consequence of the lemma proven in section 3.2 implies that there are 1 -forms $\left\{u_{\alpha}\right\}$ defined on the open sets $\left\{U_{\alpha}\right\}$ such that

$$
\zeta_{\alpha \beta}=-u_{\alpha}+u_{\beta}
$$

where

$$
u_{\alpha}=\sum \rho_{\gamma} \zeta_{\gamma \alpha}
$$

\footnotetext{
${ }^{5}$ If the strong section condition is relaxed, then the generalized transformations on the forms do not commute with the exterior derivative. So closed forms do not transform to closed forms.
} 
Thus (3.17) can be rewritten as

$$
\begin{aligned}
\left(\left(b_{\alpha}\right)_{i j}+\frac{1}{2}\left(d u_{\alpha}\right)_{i j}\right) d x_{\alpha}^{i} \wedge d x_{\alpha}^{j}= & \left(\left(b_{\beta}\right)_{i j}+\frac{1}{2}\left(d u_{\beta}\right)_{i j}\right) d x_{\beta}^{i} \wedge d x_{\beta}^{j} \\
& -\frac{1}{2} \frac{\partial}{\partial x_{\beta}^{i}}\left(u_{\alpha k} \frac{\partial x_{\alpha}^{k}}{\partial x_{\beta}^{j}}\right) d x_{\beta}^{i} \wedge d x_{\beta}^{j}+\frac{1}{2} \frac{\partial}{\partial x_{\beta}^{i}}\left(u_{\beta k} \frac{\partial x_{\alpha}^{k}}{\partial x_{\beta}^{j}}\right) d x_{\beta}^{i} \wedge d x_{\beta}^{j},
\end{aligned}
$$

or equivalently,

$$
b_{\alpha}-b_{\beta}=-\frac{1}{2} d u_{\alpha}+\frac{1}{2} d u_{\beta}-\frac{1}{2} d \tilde{u}_{\alpha}+\frac{1}{2} d \tilde{u}_{\beta}
$$

As a result

$$
\tilde{b}_{\alpha} \equiv b_{\alpha}+\frac{1}{2} d u_{\alpha}+\frac{1}{2} d \tilde{u}_{\alpha}=b_{\beta}+\frac{1}{2} d u_{\beta}+\frac{1}{2} d \tilde{u}_{\beta} \equiv \tilde{b}_{\beta} .
$$

So up to a gauge transformation, $b$ can be made globally defined on $M$ and $H=d b=d \tilde{b}$. Thus $H$ is exact.

One therefore concludes that the patching conditions induced on the spacetime from the generalized coordinate transformations of DFT after solving the strong section condition imply that $H$ is an exact form.

This conclusion cannot be satisfactory as we know string theory has as solutions spacetimes that admit non-trivial patching and a closed but not exact form $H$. The restriction that $H$ is exact is a direct consequence of the introduction of the new coordinates $y$, their transition functions and their relation to the patching conditions of the 2-form gauge potentials as this is implied from the generalized coordinate transformations.

It is worth pointing out that $H$ is not restricted to be exact in the context of generalized geometry. ${ }^{6}$ In generalized geometry, there are no additional coordinates that have to be patched. The analogous consistency condition which arises on triple overlaps reads

$$
d\left(a_{\alpha \beta}^{1}+a_{\beta \gamma}^{1}+a_{\gamma \alpha}^{1}\right)=0,
$$

and it does not impose additional restrictions on the transition functions of $H$. The above condition is always satisfied as it can be seen from the analysis of the section 3.1.

\subsection{Seeking a consistent patching}

It is not apparent how to reconcile the transition functions of the double space with those of the spacetime, and the patching conditions of the 3 -form field strength $H$ without imposing additional conditions on the fluxes. Any choice of transition functions for the double space of the type

$$
x_{\alpha}^{i}=x_{\alpha \beta}^{i}\left(x_{\beta}^{j}\right), \quad y_{\alpha i}^{1}=y_{\beta i}^{1}-\zeta_{\alpha \beta i}^{1}
$$

where the patching conditions $a_{\alpha \beta}^{1}$ of $H$ are linear combination of $\zeta_{\alpha \beta}^{1}$ 's and the combinatorial rules denoted are as those of tensor calculus, will lead to the conclusion that $H$ is exact. Another indication that such a direct approach may not be fruitful is that there is

\footnotetext{
${ }^{6}$ Generalized geometry does not require that $H$ represents a class in $H^{3}(M, \mathbb{Z})$ and so it does not capture the analogue of the Dirac quantisation condition in string theory and M-theory. It also does not obey the topological geometrisation condition unlike the $\mathrm{U}(1)$ field paradigm.
} 
nowhere use of the quantisation condition $[H] \in H^{3}(M, \mathbb{Z})$ which has a central role in the exploration of the $\mathrm{U}(1)$ paradigm.

In appendix A, a modification of the patching conditions of the $y$ coordinates was proposed and it was shown that there are no additional restrictions at triple overlaps. If this modification is considered, the double space patches consistently and it has some attractive features like its tangent bundle is an extension of the tangent bundle of the spacetime. However, the topological geometrisation of $H$ fails, i.e. when $H$ is pulled back on the double space is not exact which is in conflict with the $\mathrm{U}(1)$ field paradigm. Perhaps this is not surprising as to be able to topologically geometrize $H$, the additional coordinates have to exhibit non-trivial topology related to the class of $H$ in $H^{3}(M, \mathbb{Z})$. In the conclusions, we propose a general framework where all these questions may be addressed.

\section{Revisiting examples}

\subsection{Reviewing the double space of 3-torus with constant $H$ flux}

In this section, we review the construction of the double space of $T^{3}$ with constant $H$ flux. This example is the most relevant one of the three examples presented in [22] to explore the patching of the double spaces. This is because the consistency of the construction can be checked at the level of the transition functions for the coordinates of the double space. In the other two examples instead, the focus is on the consistency of the patching of generalized tensors.

After some relabeling of the coordinates and in geometric units, the model can be described as follows. We equip $T^{3}$ with three angular "coordinates" $7\left(\theta^{1}, \theta^{2}, \theta^{3}\right)$ such that $0 \leq \theta^{1}, \theta^{2}, \theta^{3}<2 \pi$ and take as an $H$ flux $H=N d \theta^{1} \wedge d \theta^{2} \wedge d \theta^{3}$. The quantization of flux

$$
\frac{1}{(2 \pi)^{2}} \int H \in \mathbb{Z}
$$

requires that $2 \pi N \in \mathbb{Z}$.

The construction of double space in [22] proceeds as follows. First the Poincaré lemma is used to locally solve for the gauge potential $b$ of $H$ as

$$
b=N \theta^{3} d \theta^{1} \wedge d \theta^{2} .
$$

Then it is noticed that

$$
b(2 \pi)-b(0)=2 \pi N d \theta^{1} \wedge d \theta^{2} .
$$

This apparent lack of periodicity for $b$ is compensated by a gauge transformation $b^{\prime}=b-d \xi$, where

$$
\xi=2 \pi N \theta^{1} d \theta^{2}
$$

and so

$$
b^{\prime}(2 \pi)=b(2 \pi)-2 \pi N d \theta^{1} \wedge d \theta^{2}=b(0) .
$$

\footnotetext{
${ }^{7}$ Note these are not coordinates in the sense of manifold theory. Instead they should be thought as labels that denote the points on the circle.
} 
This gauge transformation is then used to define the coordinate transformations of the DFT background as

$$
\begin{aligned}
& \theta^{\prime 1}=\theta^{1}, \quad \theta^{\prime 2}=\theta^{2}, \quad \theta^{3}=\theta^{3}, \\
& \psi^{\prime 1}=\psi^{1}, \quad \psi^{\prime 2}=\psi^{2}-2 \pi N \theta^{1}, \quad \psi^{\prime 3}=\psi^{3},
\end{aligned}
$$

according to $(3.15)$, where $\left(\psi^{1}, \psi^{2}, \psi^{3}\right)$ are the dual coordinates which are taken as the coordinates of a dual torus $\tilde{T}^{3}$.

Below we shall demonstrate that the construction of the double space relies for consistency on the existence of a particular atlas on $T^{3}$. As a result, it has limited applicability. In the process of proving this, several silent features of the above construction will become apparent.

\subsection{Patching the double space of 3-torus with constant $H$}

\subsubsection{Patching conditions}

To describe the construction of double spaces from a patching point of view, we shall first describe an atlas on the circle $S^{1}$ which in turn will induce an atlas on the 3 -torus $T^{3}$. For the former, we cover $S^{1}$ with two patches $\left\{\left(U_{1}, \varphi_{1}\right),\left(U_{2}, \varphi_{2}\right)\right\}$ such that

$$
\begin{array}{lll}
\varphi_{1}: U_{1} \subset S^{1} \rightarrow\left(-\frac{\pi}{4}, \frac{5 \pi}{4}\right), & \varphi_{1}((s, t))=x_{1}, \\
\varphi_{2}: U_{2} \subset S^{1} \rightarrow\left(-\frac{5 \pi}{4}, \frac{\pi}{4}\right), & \varphi_{2}((s, t))=x_{2},
\end{array}
$$

where $U_{1}$ is the open set which includes the north pole and $U_{2}$ is the open set which includes the south pole, and we have solved the algebraic equation $s^{2}+t^{2}=1$ of $S^{1}$ as $s=\cos x_{1}, t=\sin x_{1}$ and $s=\cos x_{2}, t=\sin x_{2}$.

The intersection $\varphi_{1}\left(U_{1} \cap U_{2}\right)=\left(-\frac{\pi}{4}, \frac{\pi}{4}\right) \cup\left(\frac{3 \pi}{4}, \frac{5 \pi}{4}\right)$ or equivalently $\varphi_{2}\left(U_{1} \cap U_{2}\right)=$ $\left(-\frac{\pi}{4}, \frac{\pi}{4}\right) \cup\left(-\frac{5 \pi}{4},-\frac{3 \pi}{4}\right)$. Therefore the transition functions are $x_{2}=\varphi_{2} \circ \varphi_{1}^{-1}\left(x_{1}\right)$ which give

$$
\begin{array}{ll}
x_{2}=x_{1}, & \text { on } \quad\left(-\frac{\pi}{4}, \frac{\pi}{4}\right), \\
x_{2}=x_{1}-2 \pi, & \text { on } \quad\left(\frac{3 \pi}{4}, \frac{5 \pi}{4}\right) .
\end{array}
$$

It is convenient to write the above transition function as

$$
x_{2}=x_{1}+2 n_{x} \pi,
$$

with the understanding that $n_{x}=0$ on $\left(-\frac{\pi}{4}, \frac{\pi}{4}\right)$ and $n_{x}=-1$ on $\left(\frac{3 \pi}{4}, \frac{5 \pi}{4}\right)$. Of course the transition function $\varphi_{1} \circ \varphi_{2}^{-1}$ is the inverse of $\varphi_{2} \circ \varphi_{1}^{-1}$, i.e. $x_{1}=x_{2}-2 n_{x} \pi$. Observe that

$$
d x_{1}=d x_{2},
$$

and so $d x$ is a globally defined closed but not exact 1-form on $S^{1}$ as expected. We refer to $x$ as the angular manifold coordinates of $S^{1}$ to distinguish them from the $\theta$ s of the previous section. 
Using the above atlas on $S^{1}$, one can induce a manifold structure on $T^{3}$ as follows. First, we introduce two patches as above for each one of the three circles, i.e. $\left\{\left(U_{i}, \varphi_{i}\right) \mid i=\right.$ $1,2\},\left\{\left(V_{j}, \lambda_{j}\right) \mid j=1,2\right\}$ and $\left\{\left(W_{k}, \mu_{k}\right) \mid k=1,2\right\}$, and then take their products. The atlas on $T^{3}$ is $\left.\left\{\left(U_{i} \times V_{j} \times W_{k}, \varphi_{i} \times \lambda_{j} \times \mu_{k}\right\}\right) \mid i, j, k=1,2\right\}$, i.e. $T^{3}$ is covered by 8 patches. It is convenient to define $U_{i j k}=U_{i} \times V_{j} \times W_{k}$ and $\varphi_{i j k}=\varphi_{i} \times \lambda_{j} \times \mu_{k}$.

In this atlas on $T^{3}$, the $H$ flux can now be written as $H=N d x \wedge d y \wedge d z$, where $x, y$ and $z$ are the angular manifold coordinates for the three circles. To continue we use the Poicaré lemma for each of the open sets $U_{i j k}$ to identify the gauge potential of $H$ as

$$
b_{i j k}=\frac{N}{3}\left(x_{i} d y \wedge d z-y_{j} d x \wedge d z+z_{k} d x \wedge d y\right) .
$$

Note that the patch Greek labels $\alpha$ of section 3.1 have been replaced with the multi-labels $i j k$. On the double overlaps $U_{i_{1} j_{1} k_{1}} \cap U_{i_{2} j_{2} k_{2}}$, we have that

$$
\begin{aligned}
b_{i_{1} j_{1} k_{1}}= & b_{i_{2} j_{2} k_{2}}+\frac{N}{3} 2 \pi\left[n_{x}\left(i_{1}-i_{2}\right) d y \wedge d z\right. \\
& \left.-n_{y}\left(j_{1}-j_{2}\right) d x \wedge d z+n_{z}\left(k_{1}-k_{2}\right) d x \wedge d y\right] .
\end{aligned}
$$

As a result, the 1 -form transition functions $a_{i_{1} j_{1} k_{1}, i_{2} j_{2} k_{2}}^{1}$ of section 3.1 can be chosen as

$$
\begin{aligned}
a_{i_{1} j_{1} k_{1}, i_{2} j_{2} k_{2}}^{1}= & \frac{N}{12} 2 \pi\left\{\left[n_{x}\left(i_{1}-i_{2}\right)\left(y_{j_{1}}+y_{j_{2}}\right)-n_{y}\left(j_{1}-j_{2}\right)\left(x_{i_{1}}+x_{i_{2}}\right)\right] d z\right. \\
& +\left[-n_{x}\left(i_{1}-i_{2}\right)\left(z_{k_{1}}+z_{k_{2}}\right)+n_{z}\left(k_{1}-k_{2}\right)\left(x_{i_{1}}+x_{i_{2}}\right)\right] d y \\
& {\left.\left[n_{y}\left(j_{1}-j_{2}\right)\left(z_{k_{1}}+z_{k_{2}}\right)-n_{z}\left(k_{1}-k_{2}\right)\left(y_{j_{1}}+y_{j_{2}}\right)\right] d x\right\} . }
\end{aligned}
$$

This expression respects the symmetries of the transition functions that arise from the exchange of the patch labels.

To continue, we compute $d a^{0}$ at triple overlaps to find that

$$
\begin{aligned}
d a_{i_{1} j_{1} k_{1}, i_{2} j_{2} k_{2}, i_{3} j_{3} k_{3}=} & \frac{N}{12}(2 \pi)^{2}\left\{n _ { x } n _ { y } \left[i_{1}\left(j_{2}-j_{3}\right)-j_{1}\left(i_{2}-i_{3}\right)-i_{2}\left(j_{1}-j_{3}\right)\right.\right. \\
& \left.+j_{2}\left(i_{1}-i_{3}\right)+i_{3}\left(j_{1}-j_{2}\right)-j_{3}\left(i_{1}-i_{2}\right)\right] d z \\
& +n_{x} n_{z}\left[-i_{1}\left(k_{2}-k_{3}\right)+i_{2}\left(k_{1}-k_{3}\right)+k_{1}\left(i_{2}-i_{3}\right)\right. \\
& \left.-k_{2}\left(i_{1}-i_{3}\right)-i_{3}\left(k_{1}-k_{2}\right)+k_{3}\left(i_{1}-i_{2}\right)\right] d y \\
& +n_{y} n_{z}\left[j_{1}\left(k_{2}-k_{3}\right)-j_{2}\left(k_{1}-k_{3}\right)-k_{1}\left(j_{2}-j_{3}\right)\right. \\
& \left.\left.+k_{2}\left(j_{1}-j_{3}\right)-k_{3}\left(j_{1}-j_{2}\right)+j_{3}\left(k_{1}-k_{2}\right)\right] d x\right\},
\end{aligned}
$$

and $n$ at quadruple intersections to find that

$$
\begin{aligned}
& n_{i_{1} j_{1} k_{1}, i_{2} j_{2} k_{2}, i_{3} j_{3} k_{3}, i_{4} j_{4} k_{4}}= \\
& \quad \frac{N}{24}(2 \pi)^{3} n_{x} n_{y} n_{z}\left\{\left[\left(i_{1} j_{2}-j_{1} i_{2}\right)\left(k_{3}-k_{4}\right)+\left(j_{1} i_{3}-j_{3} i_{1}\right)\left(k_{2}-k_{4}\right)\right.\right. \\
& \left.\quad+\left(i_{2} j_{3}-j_{2} i_{3}\right)\left(k_{1}-k_{4}\right)+\left(j_{2} i_{4}-i_{2} j_{4}\right)\left(k_{1}-k_{3}\right)+\left(j_{4} i_{1}-j_{1} i_{4}\right)\left(k_{2}-k_{3}\right)+\left(i_{3} j_{4}-j_{3} i_{4}\right)\left(k_{1}-k_{2}\right)\right] \\
& \quad-\left[\left(i_{1} k_{2}-k_{1} i_{2}\right)\left(j_{3}-j_{4}\right)+\left(k_{1} i_{3}-k_{3} i_{1}\right)\left(j_{2}-j_{4}\right)+\left(i_{2} k_{3}-k_{2} i_{3}\right)\left(j_{1}-j_{4}\right)+\left(k_{2} i_{4}-i_{2} k_{4}\right)\left(j_{1}-j_{3}\right)\right. \\
& \left.\quad+\left(k_{4} i_{1}-k_{1} i_{4}\right)\left(j_{2}-j_{3}\right)+\left(i_{3} k_{4}-k_{3} i_{4}\right)\left(j_{1}-j_{2}\right)\right]+\left[\left(j_{1} k_{2}-k_{1} j_{2}\right)\left(i_{3}-i_{4}\right)+\left(k_{1} j_{3}-k_{3} j_{1}\right)\left(i_{2}-i_{4}\right)\right. \\
& \quad+\left(j_{2} k_{3}-k_{2} j_{3}\right)\left(i_{1}-i_{4}\right)+\left(k_{2} j_{4}-k_{4} j_{2}\right)\left(i_{1}-i_{3}\right)+\left(k_{4} j_{1}-k_{1} j_{4}\right)\left(i_{2}-i_{3}\right) \\
& \left.\left.\quad+\left(j_{3} k_{4}-k_{3} j_{4}\right)\left(i_{1}-i_{2}\right)\right]\right\} .
\end{aligned}
$$


Note that $d a^{0}$ has constant components. This is an atlas dependent statement. $d a^{0}$ will not have constant components in another atlas. Of course $n$ is constant as expected independent of the choice of atlas.

Denoting the double coordinates with $X, Y, Z$, one can according to (3.15) impose the patching condition

$$
\begin{aligned}
X_{i_{1} j_{1} k_{1}} d x+Y_{i_{1} j_{1} k_{1}} d y+Z_{i_{1} j_{1} k_{1}} d z= & X_{i_{2} j_{2} k_{2}} d x+Y_{i_{2} j_{2} k_{2}} d y \\
& +Z_{i_{2} j_{2} k_{2}} d z-a_{i_{1} j_{1} k_{1}, i_{2} j_{2} k_{2}}^{1}
\end{aligned}
$$

Without further assumptions on $X, Y, Z$, a check of the consistency of the above patching condition at triple overlaps will lead to the requirement that

$$
d a^{0}=0 .
$$

This is clearly a contradiction as $d a^{0} \neq 0$ which can be seen from (4.14). This is in agreement with the proof presented in sections 3.2 and 3.3.

However for this particular example, one can also impose as patching condition

$$
\begin{gathered}
\left(X_{i_{1} j_{1} k_{1}} d x+Y_{i_{1} j_{1} k_{1}} d y+Z_{i_{1} j_{1} k_{1}} d z-X_{i_{2} j_{2} k_{2}} d x-Y_{i_{2} j_{2} k_{2}} d y\right. \\
\left.-Z_{i_{2} j_{2} k_{2}} d z+a_{i_{1} j_{1} k_{1}, i_{2} j_{2} k_{2}}^{1}\right)=0 \bmod \quad \mathbb{Z}(\ell, \ell, \ell),
\end{gathered}
$$

instead of that in (4.16). Choosing $\ell=\frac{N}{3}(2 \pi)^{2}$, the inconsistency at the triple overlaps is removed and the patching becomes consistent.

Note however that this modification of the transition functions at triple overlaps goes beyond the suggestion of [32] that the transition functions are just the local diffeomorphisms of the double space. To put it in another way, the periodic identification of the dual coordinates does not follow from the patching conditions of the double space alone as constructed from the patching conditions of $T^{3}$ and the 2 -form gauge potential. Instead it has to be imposed by hand.

It remains to investigate whether a modification of the patching condition as in (4.18) is always possible. This will be explored below.

\subsubsection{Consistency of the patching}

The modification of the patching condition as in (4.18) comes at a cost. First, the construction is atlas dependent. If one uses another atlas on $T^{3}$, the components of $d a^{0}$ are functions of the coordinates instead of constants. As a result, the modification of the patching condition as in (4.18) is not valid.

Nevertheless, one could argue that since there is an atlas on $T^{3}$ as in (4.7) and (4.8) that the patching condition (4.18) is valid, one can perform a diffeomorphism on $T^{3}$ which can take $T^{3}$ with respect to any atlas to $T^{3}$ with the (4.7) and (4.8) atlas. Such a diffeomorphism $x^{\prime}=x^{\prime}(x)$ will transform the dual coordinates schematically as

$$
X^{\prime}=\frac{\partial x}{\partial x^{\prime}} X, \quad Y^{\prime}=\frac{\partial x}{\partial x^{\prime}} Y, \quad Z^{\prime}=\frac{\partial x}{\partial x^{\prime}} Z
$$

However, such a transformation is not allowed within the DFT as does not solve the strong section condition. 
Furthermore it is worth contrasting the patching conditions (4.18) with those of (2.4) in section 2 for the 2-form paradigm. The modification of the combinatorial law in (2.4) and the subsequent consistency of patching at triple overlaps are atlas independent. As a result, the mechanism in section 2 can apply to any background and any closed 2-form provided that represents an integral class. This is not the case for the patching in (4.18) as we have seen.

The above comparison also explains the difficulty in constructing the double spaces of generic string backgrounds. If one insists of using a modification of the patching condition as in (4.18), then one has to prove that the string background admits an atlas such that at all triple overlaps $d a^{0}$ has constant components. It is not apparent that such an atlas exists for general manifolds with a closed 3 -form. So the modification of the patching conditions (4.18) may be limited to spaces with toroidal topology.

To summarize, the construction of the double space for $T^{3}$ with $H$ flux background depends on the particular atlas we have chosen on $T^{3}$. Therefore, the whole construction is rather special attached to the details of a manifold structure on $T^{3}$. Since it depends on the choice of the atlas, the construction is not general covariant and as a result (4.18) does not generalize to all spaces in a way similar to (2.4).

\section{Patching k-forms and exceptional coordinates}

\subsection{Transition functions of closed k-forms}

The patching conditions of any closed k-form $\omega^{k}, k>3$, on a manifold $M$ can be found in a similar way as those for closed 3-forms in section 3.1. For this, it is convenient to use the difference operator $\delta$ of the Čech-de Rham theory defined as

$$
\delta \chi_{\alpha_{0} \alpha_{1} \ldots \alpha_{p+1}}=\sum_{i=0}^{p+1}(-1)^{i} \chi_{\alpha_{0} \alpha_{1} \ldots \hat{\alpha}_{i} \ldots \alpha_{p+1}},
$$

where $\chi$ is a $q$-form defined at $p+1$-overlaps and the caret denotes omission. $\delta \chi$ is a $\mathrm{q}$-form defined at $(\mathrm{p}+2)$-overlaps and it is understood that in the right-hand-side of the above equation $\chi$ is restricted on $U_{\alpha_{0}} \cap \cdots \cap U_{\alpha_{p+1}}$. Observe that $\delta^{2}=0$ and $d \delta=\delta d$. For more details on the properties of $\delta$ see e.g. [41].

Given now a globally defined closed $\mathrm{k}$-form on $M, k \geq 3$, we use the Poincaré lemma to write $\omega_{\alpha}^{k}=d C_{\alpha}^{k-1}$. Then we obtain the patching conditions ${ }^{8}$

$$
\delta C_{\alpha_{0} \alpha_{1}}^{k-1}=d a_{\alpha_{0} \alpha_{1}}^{k-2}, \quad \delta a_{\alpha_{0} \alpha_{1} \alpha_{2}}^{k-2}=d a_{\alpha_{0} \alpha_{1} \alpha_{2}}^{k-3},
$$

and so on till

$$
\delta a_{\alpha_{0} \ldots \alpha_{k-1}}^{1}=d a_{\alpha_{0} \ldots \alpha_{k-1}}^{0}, \quad \delta a_{\alpha_{0} \ldots \alpha_{k}}^{0}=n_{\alpha_{0} \ldots \alpha_{k}},
$$

where $n_{\alpha_{0} \ldots \alpha_{k}}$ are constants. If $n_{\alpha_{0} \ldots \alpha_{k}} \in 2 \pi \mathbb{Z}$, then $\omega^{k}$ represents a class in $H^{k}(M, \mathbb{Z})$.

The (k-1)-form potentials $\left\{C_{\alpha}^{k-1}\right\}$ are not uniquely defined. In particular, there are defined up to a gauge transformation as

$$
C_{\alpha}^{k-1} \rightarrow C_{\alpha}^{k-1}+d \chi_{\alpha}^{k-2}
$$

\footnotetext{
${ }^{8}$ There are some sign differences in the definition of $a^{k-\ell}$ 's as compared to those in section 3.
} 
Similarly, the remaining patching conditions are defined up to gauge transformations of the type

$$
a_{\alpha_{0} \ldots \alpha_{q}}^{p} \rightarrow a_{\alpha_{0} \ldots \alpha_{q}}^{p}+d \chi_{\alpha_{0} \ldots \alpha_{q}}^{p-1}+\left(\delta \psi^{p}\right)_{\alpha_{0} \ldots \alpha_{q}}
$$

for some $\chi_{\alpha_{0} \ldots \alpha_{q}}^{p-1}$ and $\psi_{\alpha_{0} \ldots \alpha_{q-1}}^{p}$, where $p+q=k-1$. This ambiguity in the definition of patching conditions of $\omega^{k}$ is the only one allowed consistent with $d \omega^{k}=0$ and the transition functions of $M$.

\subsection{Exact k-forms and patching conditions}

As in the 3 -form case utilizing the ambiguity in the definition of the patching conditions (5.5), it is possible to show that if $a^{p}, p=0, \ldots, k-2$ satisfies the cocycle condition

$$
\left(\delta a^{p}\right)_{\alpha_{0} \ldots \alpha_{q+1}}=0
$$

then $\omega^{k}$ is exact.

In what follows, it suffices to prove this for the $a_{\alpha_{0} \alpha_{1}}^{k-2}$ as those are responsible for the patching a k-form at double overlaps, and potentially can be used to construct the exceptional generalized spaces. The proof is similar to that we have given for 3 -forms. In particular, suppose that

$$
\left(\delta a^{k-2}\right)_{\alpha_{0} \alpha_{1} \alpha_{2}}=0
$$

Then define

$$
\tilde{C}_{\alpha_{0}}^{k-1}=C_{\alpha_{0}}^{k-1}-d\left(\sum_{\gamma} \rho_{\gamma} a_{\gamma \alpha_{0}}^{k-2}\right) .
$$

Clearly $d \tilde{C}_{\alpha_{0}}^{k-1}=d C_{\alpha_{0}}^{k-1}=\omega_{\alpha_{0}}^{k}$ as $\tilde{C}^{k-1}$ and $C^{k-1}$ are related up to a gauge transformation. Moreover $\tilde{C}^{k-1}$ is a globally defined (k-1)-form as

$$
\begin{aligned}
\delta\left(\tilde{C}^{k-1}\right)_{\alpha_{0} \alpha_{1}} & =\left(\delta C^{k-1}\right)_{\alpha_{0} \alpha_{1}}-d\left(\sum_{\gamma} \rho_{\gamma}\left(a_{\gamma \alpha_{0}}^{k-2}-a_{\gamma \alpha_{1}}^{k-2}\right)\right) \\
& =d a_{\alpha_{0} \alpha_{1}}^{k-2}-d \sum_{\gamma}\left(\rho_{\gamma} a_{\alpha_{0} \alpha_{1}}^{k-2}\right)=0,
\end{aligned}
$$

where we have used (5.7) and that $\sum_{\gamma} \rho_{\gamma}=1$.

\subsection{Seeking a consistent patching for EFT}

For EFT there is not an analogue of the patching conditions of [31] available for DFT. Instead the constructions have been based on using infinitesimal symmetries generated by generalized Lie derivatives, see e.g. [13-15, 17-19, 32-34] for detailed descriptions. One expects that whatever the final form of the finite transformations are for EFT, these will generate both the transition functions of the underlying spacetime and the patching conditions of the form field strengths of the theory. After solving the strong section condition, the two must be related. Following the analogous analysis for DFT, one may hypothesize that the transition functions of the exceptional space read as

$$
x_{\alpha}^{i}=x_{\alpha \beta}\left(x_{\beta}^{j}\right), \quad y_{\alpha_{0}}^{k-2}-y_{\alpha_{1}}^{k-2}=-\zeta_{\alpha_{0} \alpha_{1}}^{k-2},
$$


where $x$ and $y^{k-2}$ are the spacetime and additional coordinates, respectively, and the patching data $a_{\alpha \beta}^{k-2}$ of the k-form field strength are a linear combination of $\zeta_{\alpha_{0} \alpha_{1}}^{k-2}$. If this is the case, then again consistency at triple overlaps will require that $\omega^{k}$ is exact.

In appendix A, we propose a modification of the patching conditions (5.10) which resolves the restriction at triple overlaps. However, it does not topologically geometrize the k-form field strength. Similar constructions can be made in theories that we include the dual fields as demonstrated in appendix A for 11-dimensional supergravity. In section 6 , we make an alternative proposal how a priori topologically geometrize k-forms field strengths based on K-theory and homotopy theory.

The above result does not hold for exceptional generalized geometries, i.e. those that no new coordinates are introduce in addition to those of spacetime. This is because they do not require the condition (5.7) but instead

$$
d \delta\left(a^{k-2}\right)_{\alpha_{0} \alpha_{1} \alpha_{2}}=0 .
$$

This does not introduce a restriction on the patching conditions of form field strengths.

\section{Summary and outlook}

We have shown that the patching conditions of DFT as arise from generalize coordinate transformations after solving the strong section condition imply that the NS-NS 3-form field is an exact 3-form. A similar conclusion may hold in the context of EFT under some plausive assumptions regarding the relation between the transition functions of additional exceptional coordinates and the patching data of the form field strengths. We have also explored some alternative possibilities. These resolve some of the difficulties, like the restriction on the form field strengths to be exact, but they do not obey the topological geometrisation condition which is one of the key properties that the $\mathrm{U}(1)$ field paradigm.

Furthermore we revisited the double space construction of $T^{3}$ with $H$-flux background from the patching conditions point of view. If the combinatorial law of the transition functions is not altered at triple overlaps, the construction is inconsistent. However, there is a modification of the combinatorial law at triple overlaps which allows for a consistent construction of the double space. But this modification depends on the choice of an atlas on $T^{3}$, i.e. it is not general covariant, and so as a result cannot be generalized to generic backgrounds with $H$-fluxes.

To introduce new coordinates that extend the spacetime in a consistent way without any further conditions on the fields, like exactness of the form field strengths, one may try to generalize some aspects of the 2-form paradigm reviewed in section 2 . Some of the directions that can be pursued are the following.

- To modify the combinatorial law of transition functions.

- To modify the transition functions.

- To introduce topology on the generalized spaces. 
It is clear that the 2-form paradigm is consistent because it has been possible to appropriately modify the combinatorial law of the transition functions. Of course, this has been achieved under the additional requirement that $\omega^{2}$ represents a class in $H^{2}(M, \mathbb{Z})$. Although this imposes a restriction on the transition functions of $\omega^{2}$, this restriction is required by the Dirac quantisation condition. In the context of DFTs and EFTs, this is an indication that non-commutative geometry has a role as it is not possible to alter the combinatorial law of transition functions in a straight forward way, see also [39, 40].

As we has seen a mild modification of the patching conditions of the form field strengths, e.g. as linear functions of the transition functions of the additional coordinates, leads to the conclusion that consistency at triple overlaps requires that the form field strengths are exact. However as we explain in appendix A, there is a modification of the transition functions of the additional coordinates such that the double and exceptional spaces are consistent at triple overlaps without any restrictions on the patching conditions of the form field strengths. However, as such a modification has its problems, like for example the topological geometrisation condition does not hold. Moreover there is no use of the cohomological analogue the Dirac quantisation condition $\left[\omega^{k}\right] \in H^{k}(M, \mathbb{Z})$ in the construction of the double of exceptional space above which has a central role in the $\mathrm{U}(1)$ field paradigm.

It is clear from the above that whatever the construction of these extended spaces is the additional coordinates have to have a non-trivial topology. This is the only way that both the topological geometrisation and the cohomological analogue of the Dirac quantisation $\left[\omega^{k}\right] \in H^{k}(M, \mathbb{Z})$ conditions can be utilized to construct these spaces. It is not a priori apparent how this can be done or whether a consistent construction is possible for all cases of interest, beyond those of toroidal compactifications, but a way to proceed is as follows.

One of the difficulties in adapting the $\mathrm{U}(1)$ field paradigm in the context of string theory and M-theory is that after applying a duality transformation the spacetime may change as a manifold at the same time as the fields of the theory. One way to incorporate this into the construction of extended spaces is as follows. Suppose that $\left(M_{I}, \mathcal{F}_{I}\right)_{I \in \mathcal{I}}$ be a family of spacetimes $M_{I}$ with field content $\mathcal{F}_{I}$ such that any two pairs $\left(M_{I}, \mathcal{F}_{I}\right)$ and $\left(M_{J}, \mathcal{F}_{J}\right)$ are related by a duality transformation $D_{I J}, D_{I J}:\left(M_{I}, \mathcal{F}_{I}\right) \rightarrow\left(M_{J}, \mathcal{F}_{J}\right)$. One way to geometrize the data $\left(M_{I}, \mathcal{F}_{I}\right)$ and $D_{I J}$ is to assume that there is a space $C M$, a C-fold, and maps $\pi_{I}: C M \rightarrow M_{I}$ such that

1. $\left(\pi_{I}\right)_{*} T C M=T M_{I}$,

2. the duality transformation $D_{I J}$ has a lift $\tilde{D}_{I J}$ on $C M$ which is implemented with a transformation which respects the topological and geometric properties of the C-fold, $C M$, and $D_{I J} \circ \pi_{I}=\pi_{J} \circ \tilde{D}_{I J}$, and

3. as a minimal requirement assert that the pull back $\pi_{I}^{*} \omega_{I}$ of all the fields $\omega_{I} \in \mathcal{F}_{I}$ on $M_{I}$ which represent non-trivial classes $\left[\omega_{I}\right]$ in cohomology $H^{*}\left(M_{I}\right)$ represent the trivial class $\left[\pi_{I}^{*} \omega_{I}\right]=0$ in $H^{*}(C M)$.

The last property is the implementation of the topological geometrisation condition which should appear as a weak restriction for carrying out the geometrisation programme. It would be necessary to impose additional conditions on $C M$ but the above three conditions can serve as a minimal requirement. 
It is not apparent whether the construction of $\mathrm{C}$-folds $C M$ would be possible for all backgrounds in string theory and M-theory. However, there are constructions which satisfy some of the three conditions mentioned above. For this consider the trivial case, where the duality transformation $D_{I J}$ is the identity and let us view $C M$ as a fibration over $M$. There are many ways that the construction of a $C M$ can be achieved which satisfies the topological geometrisation property. One way is $\mathrm{K}$-theory which generalizes the 2-form paradigm. Suppose that one tries to construct a C-fold, $C M_{\omega^{4}}$, of a spacetime that has field content a closed 4 -form $\omega^{4}$. If $\omega^{4}$ is represented by the second Chern class or the first Pontryagin class of a complex or real vector bundle $E$, respectively, then as a $C M$ can be taken as the principal bundle $P$ that $E$ is associated to. In such a case $\omega^{4}$ will represent an integral class and the pull-back of $\omega^{4}$ on $P$ will represent the trivial class. It may be possible to find amongst the K-theory class which resolves the topological geometrisation condition a representative which will also exhibit the local geometric requirements as expected from DFTs and EFTs. K-theory has appeared before in string theory and M-theory [42], see also [43] and references within. Therefore, it may not be a surprise that it could be also applicable in this context. Moreover the tangent space of all fibrations are extensions of that of the base space, and so $T\left(C M_{\omega^{4}}\right) \rightarrow \pi^{*} T M \rightarrow 0$. This is one of the properties expected for generalized manifolds.

It is tantalizing for example that one can construct C-folds for the $\mathrm{AdS}_{7} \times S^{4}$ near horizon geometry of M5-branes. It is known that for every class in $[\omega] \in H^{4}\left(S^{4}, \mathbb{Z}\right)$ there is a complex vector bundle $E$ such that $c_{2}(E)=[\omega]$. This follows from the isomorphism of $\tilde{K}\left(S^{4}\right)$ with $H^{4}\left(S^{4}, \mathbb{Z}\right)$, see e.g. [44]. The associated real bundle is an $\mathrm{SO}(4)$ bundle with Pontryangin class $p_{1}\left(E_{\mathbb{R}}\right)=2 c_{2}(E)$. In fact all the $\mathrm{SO}(4)$ bundles are classified by two integers $n, m$ as $\pi_{3}(\mathrm{SO}(4))=\mathbb{Z} \oplus \mathbb{Z}$ and $p_{1}=2(n-m)$ [45]. Considering the associated $\mathrm{SO}(4)$ principal bundles, observe that $T_{e} \mathrm{SO}(4)=\mathfrak{s o}(4)=\Lambda^{2}\left(\mathbb{R}^{4}\right)$. So the tangent space of the fibres provide a local model for the 2 -forms on $S^{4}$. This is in line with the expectation that the additional coordinates of this example are locally modeled by 2-forms on $S^{4}$.

Furthermore, there are homotopy constructions which can implement the topological geometrisation property. One such construction is the Whitehead towers, see e.g. [41]. These are given by successive fibrations

$$
K\left(\pi_{n}, n-1\right) \rightarrow Y_{n+1} \rightarrow Y_{n}
$$

where $\pi_{n}=\pi_{n}(X), Y_{1}=X, \pi_{k}\left(Y_{n}\right)=0$ for $k<n$ and $\pi_{k}\left(Y_{n}\right)=\pi_{k}(X)$ for $k>n$, and $K\left(\pi_{n}, n-1\right)$ are Eilenberg-MacLane spaces. Therefore, $Y_{2}$ is the universal cover of $X$, $\pi_{1}\left(Y_{3}\right)=\pi_{2}\left(Y_{3}\right)=0$ and so on. Thus if $[\omega]$ represents a class in the first non-vanishing cohomology group of $X$, say $H^{n}(X, \mathbb{Z})$, this class becomes trivial in $Y_{n+1}$ implementing the topological geometrisation condition.

Although the homotopy constructions above based on the Whitehead towers can implement the topological geometrisation condition, the spaces involved are infinite dimensional. It is not apparent that one can generically find a finite dimensional model which has all the required properties as for the $\mathrm{AdS}_{7} \times S^{4}$ example presented above. Nevertheless the above homotopy constructions use path spaces and so may admit a string theoretic interpretation. 
This procedure based on Whitehead towers can be applied to implement the topological geometrisation condition on a Calabi-Yau mirror pair. Suppose $X_{1}$ is a Calabi-Yau manifold and $X_{2}$ its mirror. Consider $X_{1} \times X_{2}$. The first non-vanishing cohomology group is $H^{2}\left(X_{1} \times\right.$ $\left.X_{2}\right)=H^{2}\left(X_{1}, \mathbb{Z}\right) \oplus H^{2}\left(X_{2}, \mathbb{Z}\right)$. To implement the topological geometrisation condition, it suffices to consider $Y_{4}$. By construction $H^{2}\left(Y_{4}, \mathbb{Z}\right)=H^{3}\left(Y_{4}, \mathbb{Z}\right)=0$ and $Y_{4}$ fibres above $X_{1} \times X_{2}$ and so above both $X_{1}$ and $X_{2}$. Therefore the $H^{2}$ and $H^{3}$ cohomologies classes of both $X_{1}$ and $X_{2}$ become trivial in $Y_{4}$. It will be of interest to determine the action of $\tilde{D}_{12}$ on $Y_{4}$, where $D_{12}$ is the mirror symmetry of $X_{1}$ and $X_{2}$.

Therefore there are some suggestions how to construct generalized manifolds which allow for form field strengths to represent non-trivial cohomology classes. However, it is less apparent which will be the most fruitful way to proceed. The expectation is that the full theory at the end will combine many of the local computations that have been done so far with the global aspects that many of the backgrounds have in a consistent way.

\section{Acknowledgments}

I would like to thank Martin Cederwall, Ulf Gran and specially David Berman for many helpful comments. I am partially supported by the STFC grant ST/J002798/1.

Note added. Part of the title of this paper was inspired from an article by Greg Child contained in his book "Mixed Emotions", published by The Mountaineers, Seattle Washington (1993).

\section{A Modifying transition functions}

\section{A.1 New transition functions and k-forms}

Let $\omega^{k}$ be closed k-form on $M, k \geq 3$, with transition functions as those defined in section 4 . To define $\tilde{M}_{\omega^{k}}$, we introduce coordinates $\left(x, y^{k-2}\right)$, where $x$ are the coordinates of $M$, and impose the transition functions

$$
x_{\alpha_{0}}=f_{\alpha_{0} \alpha_{1}}\left(x_{\alpha_{1}}\right), \quad \delta y_{\alpha_{0} \alpha_{1}}^{k-2} \equiv-y_{\alpha_{0}}^{k-2}+y_{\alpha_{1}}^{k-2}=a_{\alpha_{0} \alpha_{1}}^{k-2}-\sum_{\beta} \rho_{\beta} d a_{\beta \alpha_{0} \alpha_{1}}^{k-3}
$$

Since $\delta^{2}=0$, consistency at triple overlaps requires that

$$
\delta\left(a^{k-2}-\sum_{\beta} \rho_{\beta} d a_{\beta}^{k-3}\right)_{\alpha_{0} \alpha_{1} \alpha_{2}}=0
$$

which is satisfied as it can be shown after a calculation using (5.2).

It is clear that there is a projection $\pi$ from $\tilde{M}_{\omega^{k}}$ onto $M$ and so it can be thought that $\tilde{M}_{\omega^{k}}$ is a bundle over $M$. However as in the case of 3 -forms there is no natural global section of $M$ in $\tilde{M}_{\omega^{k}}$, e.g. the zero section is not preserved by the transition functions. 
Since $\tilde{M}_{\omega^{k}}$ is a manifold, one can investigate the tangent as well as all the other tensor bundles in the standard way. We shall do this for $\tilde{M}_{\omega^{3}}$ as the generalization to $\tilde{M}_{\omega^{k}}$ is straightforward. To do this let us write the patching conditions (5.10) explicitly as

$$
x_{\alpha}^{i}=f_{\alpha \beta}^{i}\left(x_{\beta}^{j}\right), \quad\left(y_{\alpha}^{1}\right)_{i}-\left(y_{\beta}^{1}\right)_{k} \frac{\partial x_{\beta}^{k}}{\partial x_{\alpha}^{i}}=\left(\tilde{a}_{\alpha \beta}^{1}\right)_{i}
$$

where

$$
\tilde{a}_{\alpha \beta}^{1}=-a_{\alpha \beta}^{1}+\Sigma_{\delta} \rho_{\delta} d a_{\delta \alpha \beta}^{0} .
$$

Note that the second patching condition in (A.3) does not satisfy the strong section condition. However one can do a coordinate redefinition and set simply $\left(y_{\alpha}^{1}\right)_{i}=\left(y_{\beta}^{1}\right)_{i}+\left(\tilde{a}_{\alpha \beta}^{1}\right)_{i}$ but this is less attractive in calculations.

To find the patching conditions of the tangent bundle consider the vector field $X_{\alpha}=$ $A_{\alpha}^{i} \frac{\partial}{\partial x_{\alpha}^{i}}+B_{\alpha, i} \frac{\partial}{\partial\left(y_{\alpha}\right)_{i}}$ and demanding $X_{\alpha}=X_{\beta}$, we find that

$$
\begin{aligned}
A_{\alpha}^{i} & =\frac{\partial x_{\alpha}^{i}}{\partial x_{\beta}^{j}} A_{\beta}^{j}, \\
\left(B_{\alpha}\right)_{i} & =\frac{\partial x_{\beta}^{k}}{\partial x_{\alpha}^{i}}\left(B_{\beta}\right)_{k}+\frac{\partial x_{\alpha}^{m}}{\partial x_{\beta}^{p}} \frac{\partial^{2} x_{\beta}^{k}}{\partial x_{\alpha}^{m} \partial x_{\alpha}^{i}}\left(y_{\beta}\right)_{k} A_{\beta}^{p}+\frac{\partial\left(\tilde{a}_{\alpha \beta}^{1}\right)_{i}}{\partial x_{\beta}^{k}} A_{\beta}^{k} .
\end{aligned}
$$

It is clear from this that $T \tilde{M}_{\omega^{3}}$ is an extension of $\pi^{*} T M$ with respect to the cotangent bundle $\pi^{*} \Lambda^{1} M$ of $M$, i.e.

$$
0 \rightarrow \pi^{*} \Lambda^{1} M \rightarrow T \tilde{M}_{\omega^{3}} \rightarrow \pi^{*} T M \rightarrow 0 .
$$

In particular, $\pi^{*} \Lambda^{1} M$ is a subbundle of $T \tilde{M}_{\omega^{3}}$. This is reminiscent of generalized geometry where the bundle $E$ over $M$ which is an extension of $T M$ is now replaced with $T \tilde{M}_{\omega^{3}}$.

Furthermore observe that the pairing of $T M$ and $\Lambda^{1} M$ is naturally extended to $T \tilde{M}_{\omega^{3}}$ as the transition functions of $T \tilde{M}_{\omega^{3}}$ preserve it. As a result, one can define an $O(n, n)$ structure on $T \tilde{M}_{\omega^{3}}$ as expected from string theory considerations which also arises in the context of generalized geometry. But of course this $O(n, n)$ is related to the modified transition functions rather than those associated to the original patching conditions $a_{\alpha \beta}^{1}$ of of the 2-form gauge potential.

In the more general case of $\tilde{M}_{\omega^{k}}$, one finds that

$$
0 \rightarrow \pi^{*} \Lambda^{k-2} M \rightarrow T M_{\omega^{k}} \rightarrow \pi^{*} T M \rightarrow 0 .
$$

\section{A.2 Testing for other properties}

One of the properties of the construction of C-folds for 2 -forms $\omega^{2}$ is that when one pulls back the 2-form on the C-fold, $\omega^{2}$ becomes exact. It is not expected that this property holds on $\tilde{M}_{\omega^{k}}$ because by construction the fibres have trivial topology, i.e. by construction $\tilde{M}_{\omega^{k}}$ is contractible to $M$. Nevertheless, it is instructive to see what the result is. In analogy with the 2-form case, we take the exterior derivative of second transition function in (5.10) to find

$$
d y_{\alpha}^{k-2}-d y_{\beta}^{k-2}=C_{\alpha}^{k-1}-C_{\beta}^{k-1}+\Sigma_{\gamma} d \rho_{\gamma} \wedge d a_{\alpha \beta \gamma}^{k-3}
$$


Using the properties of the partition of unity and the patching conditions of $\omega^{k}$, this can be rewritten as

$$
d y_{\alpha}^{k-2}-C_{\alpha}^{k-1}-\sum_{\gamma} d \rho_{\gamma} \wedge a_{\gamma \alpha}^{k-2}=d y_{\beta}^{k-2}-C_{\beta}^{k-1}-\sum_{\gamma} d \rho_{\gamma} \wedge a_{\gamma \beta}^{k-2} .
$$

It is clear from the above equation that $\omega^{k}$ cannot be written as the exterior derivative of a $(k-1)$-form on $\tilde{M}_{\omega^{k}}$. For the latter, one would have expected that $d y_{\alpha}^{k-2}-C_{\alpha}^{k-1}$ should have patched globally as a (k-1)-form on $\tilde{M}_{\omega^{k}}$. But as we have shown this is not the case and the (k-1)-form that patches globally receives a correction that depends on the derivative of the functions $\rho_{\alpha}$ which appear in the partition of unity. This correction is like a source term. Assuming that the partition of unity functions have compact support and that the good cover is very fine, $\rho_{\alpha}$ resemble delta-functions. Therefore the source term is like the derivative of a delta function. Note that in the construction of some exceptional field theories additional field are required in the form of tensor hierarchies, see [17-19] which is reminiscent to these additional terms.

\section{A.3 $\tilde{M}$ for M-theory}

It would be of interest in view of applications in strings and M-theory to generalize the construction of the previous appendix from single k-forms, $\omega^{k}$, to differential algebras $\mathcal{A}$. We shall not give a general treatment of this. Instead we shall focus on the differential algebra of M-theory generated by the 4-form field strength $F$ and its dual $G$, where now

$$
\mathcal{A}: \quad d F=0, \quad d G=F \wedge F,
$$

and $G$ is treated as an independent field.

Suppose that $M$ now has a good cover, the above equations can be solved at each open set of the cover as

$$
F_{\alpha}=d C_{\alpha}^{3}, \quad G_{\alpha}=d C_{\alpha}^{6}+C_{\alpha}^{3} \wedge F_{\alpha} .
$$

Next on double overlaps, we have

$$
\delta C_{\alpha \beta}^{3}=d a_{\alpha \beta}^{2}, \quad \delta C_{\alpha \beta}^{6}=d b_{\alpha \beta}^{5}-a_{\alpha \beta}^{2} \wedge F
$$

and at triple overlaps

$$
\delta a_{\alpha \beta \gamma}^{2}=d a_{\alpha \beta \gamma}^{1}, \quad \delta b_{\alpha \beta \gamma}^{5}=d b_{\alpha \beta \gamma}^{4}+a_{\alpha \beta \gamma}^{1} \wedge F .
$$

Next to construct $\tilde{M}$, we introduce coordinates $\left(x, y^{2}, w^{5}\right)$ and introduce the transition functions

$$
\begin{aligned}
x_{\alpha} & =f_{\alpha \beta}\left(x_{\beta}\right), \quad y_{\alpha}^{2}-y_{\beta}^{2}=a_{\alpha \beta}^{2}-\sum_{\gamma} \rho_{\gamma} d a_{\gamma \alpha \beta}^{1}, \\
w_{\alpha}^{5}-w_{\beta}^{5} & =b_{\alpha \beta}^{5}-\sum_{\gamma} \rho_{\gamma}\left(a_{\gamma \alpha \beta}^{1} \wedge F+d b_{\gamma \alpha \beta}^{4}\right) .
\end{aligned}
$$


After performing a computation similar to that we have explained in previous case, the transition functions are consistent at triple overlaps. This proves that one can define a manifold $\tilde{M}_{\mathcal{A}}$ for the M-theory differential algebra $\mathcal{A}$ in (A.10).

Most of the properties of the $\tilde{M}_{\omega^{k}}$ spaces constructed for single k-forms can be generalized to this case. First there is a projection from $\tilde{M}_{\mathcal{A}}$ onto $M$, and so $\tilde{M}_{\mathcal{A}}$ can be thought as a bundle over $M$. The tangent space $T \tilde{M}_{\mathcal{A}}$ is an extension of $T M$ with respect to the space of 2 - and 5 -forms on $M$, i.e.

$$
0 \rightarrow \pi^{*} \Lambda^{2} M \oplus \pi^{*} \Lambda^{5} M \rightarrow T \tilde{M}_{\mathcal{A}} \rightarrow \pi^{*} T M \rightarrow 0
$$

As this construction is intended as an application to M-theory, $M$ is 11-dimensional. However a similar construction can be applied to compactifications.

Open Access. This article is distributed under the terms of the Creative Commons Attribution License (CC-BY 4.0), which permits any use, distribution and reproduction in any medium, provided the original author(s) and source are credited.

\section{References}

[1] M.J. Duff, Duality Rotations in String Theory, Nucl. Phys. B 335 (1990) 610 [inSPIRE].

[2] A.A. Tseytlin, Duality symmetric closed string theory and interacting chiral scalars, Nucl. Phys. B 350 (1991) 395 [inSPIRE].

[3] W. Siegel, Two vierbein formalism for string inspired axionic gravity, Phys. Rev. D 47 (1993) 5453 [hep-th/9302036] [INSPIRE].

[4] W. Siegel, Superspace duality in low-energy superstrings, Phys. Rev. D 48 (1993) 2826 [hep-th/9305073] [INSPIRE].

[5] C.M. Hull, Doubled Geometry and T-Folds, JHEP 07 (2007) 080 [hep-th/0605149] [INSPIRE].

[6] O. Hohm, C. Hull and B. Zwiebach, Background independent action for double field theory, JHEP 07 (2010) 016 [arXiv: 1003.5027] [INSPIRE].

[7] O. Hohm, C. Hull and B. Zwiebach, Generalized metric formulation of double field theory, JHEP 08 (2010) 008 [arXiv: 1006 .4823] [INSPIRE].

[8] I. Jeon, K. Lee and J.-H. Park, Differential geometry with a projection: Application to double field theory, JHEP 04 (2011) 014 [arXiv: 1011.1324] [INSPIRE].

[9] I. Jeon, K. Lee and J.-H. Park, Stringy differential geometry, beyond Riemann, Phys. Rev. D 84 (2011) 044022 [arXiv: 1105.6294] [INSPIRE].

[10] O. Hohm and S.K. Kwak, Frame-like Geometry of Double Field Theory, J. Phys. A 44 (2011) 085404 [arXiv: 1011.4101] [INSPIRE].

[11] P.C. West, $E_{11}$ and M-theory, Class. Quant. Grav. 18 (2001) 4443 [hep-th/0104081] [INSPIRE].

[12] F. Englert, L. Houart, A. Taormina and P.C. West, The symmetry of $M$ theories, JHEP 09 (2003) 020 [hep-th/0304206] [INSPIRE]. 
[13] D.S. Berman, H. Godazgar, M. Godazgar and M.J. Perry, The local symmetries of M-theory and their formulation in generalised geometry, JHEP 01 (2012) 012 [arXiv:1110.3930] [INSPIRE].

[14] A. Coimbra, C. Strickland-Constable and D. Waldram, $E_{d(d)} \times \mathbb{R}^{+}$generalised geometry, connections and M-theory, JHEP 02 (2014) 054 [arXiv:1112.3989] [INSPIRE].

[15] A. Coimbra, C. Strickland-Constable and D. Waldram, Supergravity as Generalised Geometry II: $E_{d(d)} \times \mathbb{R}^{+}$and M-theory, JHEP 03 (2014) 019 [arXiv:1212.1586] [INSPIRE].

[16] H. Godazgar, M. Godazgar and H. Nicolai, Generalised geometry from the ground up, JHEP 02 (2014) 075 [arXiv: 1307.8295] [INSPIRE].

[17] O. Hohm and H. Samtleben, Exceptional Field Theory I: $E_{6(6)}$ covariant Form of M-theory and Type IIB, Phys. Rev. D 89 (2014) 066016 [arXiv:1312.0614] [InSPIRE].

[18] O. Hohm and H. Samtleben, Exceptional Field Theory II: E $E_{7(7)}$, Phys. Rev. D 89 (2014) 066017 [arXiv:1312.4542] [InSPIRE].

[19] G. Aldazabal, M. Graña, D. Marqués and J.A. Rosabal, The gauge structure of Exceptional Field Theories and the tensor hierarchy, JHEP 04 (2014) 049 [arXiv:1312.4549] [INSPIRE].

[20] G. Aldazabal, D. Marques and C. Núñez, Double Field Theory: A Pedagogical Review, Class. Quant. Grav. 30 (2013) 163001 [arXiv:1305.1907] [INSPIRE].

[21] D.S. Berman and D.C. Thompson, Duality Symmetric String and M-theory, arXiv: 1306.2643 [INSPIRE].

[22] O. Hohm, D. Lüst and B. Zwiebach, The Spacetime of Double Field Theory: Review, Remarks and Outlook, Fortsch. Phys. 61 (2013) 926 [arXiv:1309.2977] [INSPIRE].

[23] B. de Wit and H. Nicolai, $d=11$ Supergravity With Local SU(8) Invariance, Nucl. Phys. B 274 (1986) 363 [InSPIRE].

[24] N. Hitchin, Generalized Calabi-Yau manifolds, Quart. J. Math. Oxford Ser. 54 (2003) 281 [math/0209099] [INSPIRE].

[25] N. Hitchin, Brackets, forms and invariant functionals, math/0508618 [INSPIRE].

[26] M. Gualtieri, Generalized complex geometry, math/0401221 [INSPIRE].

[27] C.M. Hull, Generalised Geometry for M-theory, JHEP 07 (2007) 079 [hep-th/0701203] [INSPIRE].

[28] P.P. Pacheco and D. Waldram, M-theory, exceptional generalised geometry and superpotentials, JHEP 09 (2008) 123 [arXiv:0804.1362] [INSPIRE].

[29] C. Hillmann, Generalized $E(7(7))$ coset dynamics and $D=11$ supergravity, JHEP 03 (2009) 135 [arXiv:0901.1581] [INSPIRE].

[30] D.S. Berman and M.J. Perry, Generalized Geometry and M-theory, JHEP 06 (2011) 074 [arXiv: 1008.1763] [INSPIRE].

[31] O. Hohm and B. Zwiebach, Large Gauge Transformations in Double Field Theory, JHEP 02 (2013) 075 [arXiv: 1207.4198] [INSPIRE].

[32] D.S. Berman, M. Cederwall and M.J. Perry, Global aspects of double geometry, JHEP 09 (2014) 066 [arXiv: 1401.1311] [INSPIRE].

[33] D.S. Berman, M. Cederwall, A. Kleinschmidt and D.C. Thompson, The gauge structure of generalised diffeomorphisms, JHEP 01 (2013) 064 [arXiv:1208.5884] [INSPIRE]. 
[34] M. Cederwall, J. Edlund and A. Karlsson, Exceptional geometry and tensor fields, JHEP 07 (2013) 028 [arXiv: 1302.6736] [INSPIRE].

[35] I. Vaisman, Towards a double field theory on para-Hermitian manifolds, J. Math. Phys. 54 (2013) 123507 [arXiv:1209.0152] [INSPIRE].

[36] T. Kikuchi, T. Okada and Y. Sakatani, Rotating string in doubled geometry with generalized isometries, Phys. Rev. D 86 (2012) 046001 [arXiv: 1205.5549] [INSPIRE].

[37] H. Godazgar, M. Godazgar and H. Nicolai, Einstein-Cartan Calculus for Exceptional Geometry, JHEP 06 (2014) 021 [arXiv:1401.5984] [INSPIRE].

[38] E. Cartan, Les groupes des transformations continus, infinis, simples, Ann. Sci. Ecole Norm. Sup. 26 (1909) 93.

[39] I. Bakas and D. Lüst, 3-Cocycles, Non-Associative Star-Products and the Magnetic Paradigm of R-Flux String Vacua, JHEP 01 (2014) 171 [arXiv: 1309.3172] [INSPIRE].

[40] R. Blumenhagen, M. Fuchs, F. Haßler, D. Lüst and R. Sun, Non-associative Deformations of Geometry in Double Field Theory, JHEP 04 (2014) 141 [arXiv:1312.0719] [INSPIRE].

[41] R. Bott and L.W. Tu, Differential forms in algebraic topology, Springer-Verlag, Graduate Texts in Mathematics $\mathbf{8 2}$ (1982).

[42] E. Witten, D-branes and k-theory, JHEP 12 (1998) 019 [hep-th/9810188] [INSPIRE].

[43] D.-E. Diaconescu, G.W. Moore and E. Witten, A derivation of k-theory from $M$-theory, hep-th/0005091 [INSPIRE].

[44] D. Husemoller, Fibre Bundles, Springer-Verlag, Graduate Texts in Mathematics 20 (1966).

[45] J. Milnor, On manifolds homeomorphic to the 7-sphere, Ann. Math. 64 (1959) 399. 